\title{
Association between physical fitness and b-vitamin status in Spanish elderly people
}

Physical fitness (PF) is associated as an independent predictor of all-cause diseases and mortality ${ }^{(1,2)}$. On the other hand, low concentrations of b-vitamin are prevalent with advancing age. Furthermore, some authors suggest total homocysteine (tHcy) seem to be a functional indicator of these vitamins deficiencies ${ }^{(3)}$.The aim of this study was to assess the association between PF, b-vitamin status and tHcy levels in Spanish population over 55 years. Battery of 4 PF tests was applied to a sub-sample of a PREDIMED study (427 participants, $57 \%$ women, 55-88 years). The score for each test was from 0 (worst) to 3 (best result) points. Subjects were classified in two groups according to total scores: i) low fitness: 0-8; and ii) high fitness: 9-12 points, stratified by gender. Blood samples were collected. Data were analyzed using one-way ANOVA.

Table 1. Vitamin B and homocysteine levels by different level of fitness.

\begin{tabular}{|c|c|c|c|c|c|c|}
\hline & \multicolumn{2}{|c|}{ Male $(n=129 / 55)$} & \multirow[b]{2}{*}{$\mathbf{p}$} & \multicolumn{2}{|c|}{ Female $(n=173 / 70)$} & \multirow[b]{2}{*}{$\mathrm{p}$} \\
\hline & Mean & SD & & Mean & SD & \\
\hline \multicolumn{7}{|c|}{ tHcy $(\mu \mathrm{mol} / \mathrm{dl})$} \\
\hline Low fitness & $15 \cdot 08$ & $4 \cdot 79$ & \multirow[t]{2}{*}{$0 \cdot 021$} & 11.79 & 3.71 & \multirow[t]{2}{*}{0.521} \\
\hline High fitness & $13 \cdot 43$ & $3 \cdot 29$ & & 11.42 & $4 \cdot 37$ & \\
\hline \multicolumn{7}{|c|}{ Vitamin $B_{12}(\mathrm{ng} / \mathrm{mL})$} \\
\hline Low fitness & $347 \cdot 73$ & $151 \cdot 11$ & \multirow[t]{2}{*}{$0 \cdot 558$} & $478 \cdot 15$ & $605 \cdot 70$ & \multirow[t]{2}{*}{0.524} \\
\hline High fitness & $333 \cdot 31$ & $122 \cdot 81$ & & $430 \cdot 15$ & $194 \cdot 64$ & \\
\hline \multicolumn{7}{|c|}{ Serum folic acid $(\mu \mathrm{g} / \mathrm{mL})$} \\
\hline Low fitness & 9.97 & 3.97 & \multirow[t]{2}{*}{$0 \cdot 034$} & $11 \cdot 17$ & $4 \cdot 52$ & \multirow[t]{2}{*}{$0 \cdot 093$} \\
\hline High fitness & $11 \cdot 33$ & 3.83 & & $12 \cdot 27$ & $4 \cdot 47$ & \\
\hline \multicolumn{7}{|c|}{ RBC folate (ng/ mL) } \\
\hline Low fitness & $376 \cdot 2$ & $109 \cdot 7$ & \multirow[t]{2}{*}{$0 \cdot 374$} & $351 \cdot 9$ & $119 \cdot 6$ & \multirow[t]{2}{*}{$0 \cdot 213$} \\
\hline High fitness & $395 \cdot 0$ & $84 \cdot 3$ & & $374 \cdot 7$ & $121 \cdot 1$ & \\
\hline
\end{tabular}

Data are expressed by mean and standard deviation (SD). tHcy: total homocysteine; RBC: red blood cell folate. Data are controlled by age, city and total METs/hour.

The table 1 shows serum folic acid and tHcy in males was significant better $(\mathrm{p}<0.05)$ in those who had high PF level than those had low PF. However, there were not significant differences in all biomarkers in females. In conclusion, males with high physical fitness have shown better serum folic acid and tHcy levels than low physical fitness. Supported by Instituto Salud Carlos III (PI11/01791).

1.Pedrero-Chamizo R, Gomez-Cabello A, et al. Physical fitness levels among independent non-institutionalized Spanish elderly: the elderly EXERNET multi-center study. Arch Gerontol Geriatr. 2012;55(2):406-16.

2. Swift DL, Lavie CJ, Johannsen NM, et al. Physical activity, cardiorespiratory fitness, and exercise training in primary and secondary coronary prevention. Circ J. 2013;77(2):281-92.

3. De Jong N, Chin APMJ, et al. Nutrient-dense foods and exercise in frail elderly: effects on B vitamins, hcy, methylmalonic acid, and neuropsychological functioning. Am J Clin Nutr. 2001;73(2):338-46. 